\title{
designGG: an R-package and web tool for the optimal design of genetical genomics experiments
}

\author{
Yang Li*1, Morris A Swertz ${ }^{1,2}$, Gonzalo Vera1, Jingyuan Fu², Rainer Breitling ${ }^{1}$ \\ and Ritsert C Jansen ${ }^{1,2}$
}

Address: ${ }^{1}$ Groningen Bioinformatics Center, Groningen Biomolecular Sciences and Biotechnology Institute, University of Groningen, Haren, The Netherlands and 2Department of Genetics, University Medical Center Groningen and University of Groningen, Groningen, The Netherlands

Email: Yang Li* - yang.li@rug.nl; Morris A Swertz - m.a.swertz@rug.nl; Gonzalo Vera - gonzalo.vera.rodriguez@gmail.com; Jingyuan Fu - j.fu@rug.nl; Rainer Breitling - r.breitling@rug.nl; Ritsert C Jansen - r.c.jansen@rug.nl

* Corresponding author

Published: 18 June 2009

BMC Bioinformatics 2009, 10:188 doi:10.1186/1471-2105-10-188
Received: 23 April 2009

Accepted: 18 June 2009

This article is available from: http://www.biomedcentral.com/I47I-2/05//0/I88

(c) 2009 Li et al; licensee BioMed Central Ltd.

This is an Open Access article distributed under the terms of the Creative Commons Attribution License (http://creativecommons.org/licenses/by/2.0), which permits unrestricted use, distribution, and reproduction in any medium, provided the original work is properly cited.

\begin{abstract}
Background: High-dimensional biomolecular profiling of genetically different individuals in one or more environmental conditions is an increasingly popular strategy for exploring the functioning of complex biological systems. The optimal design of such genetical genomics experiments in a costefficient and effective way is not trivial.

Results: This paper presents designGG, an $\mathrm{R}$ package for designing optimal genetical genomics experiments. A web implementation for designGG is available at http://gbic.biol.rug.nl/designGG. All software, including source code and documentation, is freely available.

Conclusion: DesignGG allows users to intelligently select and allocate individuals to experimental units and conditions such as drug treatment. The user can maximize the power and resolution of detecting genetic, environmental and interaction effects in a genome-wide or local mode by giving more weight to genome regions of special interest, such as previously detected phenotypic quantitative trait loci. This will help to achieve high power and more accurate estimates of the effects of interesting factors, and thus yield a more reliable biological interpretation of data. DesignGG is applicable to linkage analysis of experimental crosses, e.g. recombinant inbred lines, as well as to association analysis of natural populations.
\end{abstract}

\section{Background}

Genetical genomics [1] has become a popular strategy for studying complex biological systems using a combination of classical genetics, biomolecular profiling and bioinformatics [2-5]. By measuring molecular variation, using transcriptomics, proteomics, metabolomics and related emerging technologies, in genetically different individuals, genetical genomics has the potential to identify the functional consequences of natural and induced genetic variation. Recently, genetical genomics has been generalized to achieve a comprehensive understanding of the dynamics of molecular networks by combining environmental and genetic perturbation [6,7]. This type of large scale "omics" study leads to a better understanding of why individuals of the same species respond differently to drugs, pathogens, and other environmental factors. 


\section{Optimize your Genetical Genomics Experiment}

1. Define analysis platform (2)

- Single channel

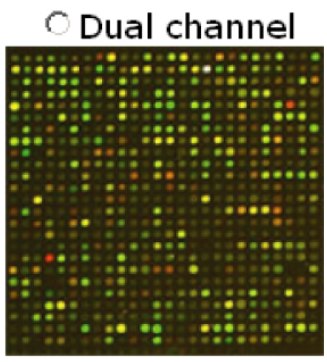

2. Define individual genotypes (2)

Upload tab delimited file, e.g.

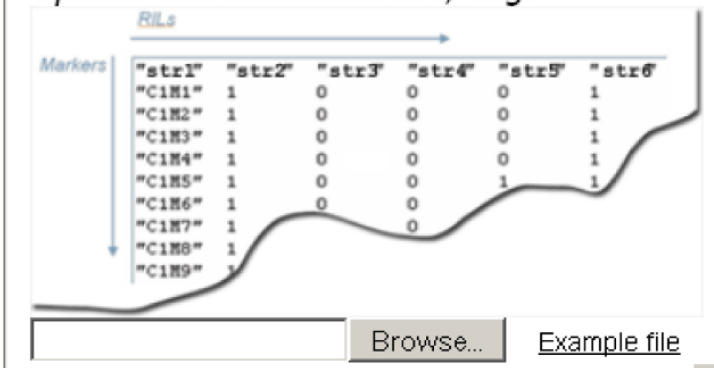

3. Define experimental factors 0

\begin{tabular}{|c|c|c|c|c|c|}
\hline Factors & Leve & & & & \\
\hline$\sqrt{\text { TempCelsius }}$ & 15 & 24 & 29 & + & - \\
\hline$\sqrt{\nabla}$ Tissue & Brain & Liver & Kidney & + & - \\
\hline$\Gamma \longdiv { \text { Factor } 3 }$ & 1 & 2 & 3 & + & -1 \\
\hline
\end{tabular}

4. Set constraints

Total number of slides

- Number of strains per level 4

\section{Optimize Experiment Design Test}

Figure I

Screenshot of the designGG web interface. 
Table I: Example table of genotype data. Heterozygous loci are indicated by an $\mathbf{H}$.

\begin{tabular}{lllllll}
\hline & Strain I & Strain 2 & Strain 3 & Strain 4 & Strain 5 & $\ldots$ \\
\hline CIMI & A & B & B & B & A & $\ldots$ \\
\hline CIM2 & A & H & A & B & A & $\ldots$ \\
\hline CIM3 & A & A & B & H & A & $\ldots$ \\
\hline$\ldots$ & $\ldots$ & $\ldots$ & $\ldots$ & $\ldots$ & $\ldots$ & $\ldots$
\end{tabular}

However, most molecular profiling experiments are very costly, and as a consequence most genetical genomics studies are performed at the verge of statistical feasibility. Therefore, experimental design needs careful consideration to achieve maximum power from limited resources, such as microarrays and experimental animals $[8,9]$. But, even in standard scenarios this requires sophisticated application of statistical concepts to intelligently select genetically different individuals from a population and allocate them to different conditions and experimental units. This topic has motivated classical statistical research since a long time [10]. More recently, the concepts developed there have been adapted to the high dimensional data sets of post-genomics research [8,11-13], and useful simplified design strategies have been suggested $[11,14]$. However, to transfer these statistical ideas to the even more complex context of genetical genomics $[9,15,16]$ still requires considerable expertise in statistics.

Here we present an online web tool to make these selections and allocations easy for biologists with little/no statistical training. The program will find the best experimental design to produce the most accurate estimates of the most relevant biological parameters, given the number of experimental factors to be varied, the genotype information on the population, the profiling technology used, and the constraints on the number of individuals that can be profiled. Advanced users can download the underlying methods as an $\mathrm{R}$ package to adapt the program for a more tailored design. Without loss of generality, we will illustrate the method using microarrays, while they apply equally well to other profiling technologies, such as mass spectrometry. Also, we will only discuss molecular technologies that profile samples individually (e.g., single color microarrays) or in pairs (e.g., dual color microarrays), but an extension of the $\mathrm{R}$ scripts to more advanced multiplex technologies would be straightforward [17].

\section{Implementation}

The objective of designGG is to find an optimal allocation of genetically different samples to different conditions and experimental units (arrays) favoring a precise esti- mate of interesting parameters, such as main genetic effects and interaction effects between genotype and drug treatment. A simple case with one environmental factor can be expressed as $y=\mu+\mathrm{G} \times \mathrm{E}+\varepsilon$, where $\mathrm{y}$ is the measurement vector, $\varepsilon$ is the error term, and $\mathrm{G} \times \mathrm{E}$ denotes main effect and interaction effects of genotype and environment. In matrix notation, a model with one or more genotype factors (quantitative trait loci; QTL) and one or more environmental factors can be written as: $\mathbf{Y}=\mathbf{X} \beta+\mathrm{E}$, where $\mathbf{X}$ is the design matrix of samples by parameters and $\beta$ is the effect of genotype and environmental factors. The least squares estimate of $\beta$ is $b=\left(X^{\mathrm{T}} \mathbf{X}\right)^{-1} \mathbf{X}^{\mathrm{T}} \mathbf{Y}$ with $\operatorname{var}(\mathrm{b})=$ $\sigma^{2}\left(\mathbf{X}^{\mathrm{T}} \mathbf{X}\right)^{-1}$. The optimal experiment design is defined as the one that minimizes the double sum of the variances of b firstly summed over all parameters and then summed over all genotypic markers. We use an optimization algorithm (simulated annealing [18]) to search the experimental design space of all possible allocations to produce an optimal design matrix $\mathbf{X}$. During the optimization, the algorithm utilizes the available marker information from the individuals to optimize the allocation of individuals to microarrays and conditions.

In the optimization, the experimenter can, of course, give more weight to parameters of higher interest, which will then be estimated with higher accuracy. Particularly, prior knowledge about expected effect sizes of interesting factors can be incorporated as weight parameters for the algorithm and the weight is inversely proportional to the expected effect size of the corresponding factors. In addition, it is also possible to specify the genome regions that are of major interest in a particular experiment, by specifying a region parameter. For example, if the relevant phenotype is known to map to certain genome regions, parameters for the markers in these regions can be given full weight in the optimization algorithm, whereas parameters for other markers can be given lesser or even zero weight. Thus, mapping resolution can improve and the power for finding QTLs in focal regions can be increased.

DesignGG is a package entirely written in the R language [19]. Every function of the designGG library is available as a stand-alone R tool and detailed help is available according to the standard format of $\mathrm{R}$ documentation.

\section{Results \\ Web tool}

Users can apply this method using a web interface (Figure 1 ) that we have generated using MOLGENIS $[20,21]$ :

1. Choose the platform. Select the single- or dual-channel option for one-color or two-color gene expression microarrays (the dual-channel option is also used for any other technology profiling pairs of samples). 
Table 2: The description and possible values of designGG arguments

\begin{tabular}{|c|c|c|}
\hline Arguments & Description & Possible value(s) \\
\hline bTwoColorArraya & The type of platform & $\begin{array}{l}\mathrm{T} \text { ( RUE ) or } \mathrm{F} \text { ( } \mathrm{ALSE} \text { ) for the dual- or single-channel option, } \\
\text { respectively. For example, } \mathrm{F} \text { for one-color and } \mathrm{T} \text { for two-color } \\
\text { gene expression microarrays } \\
\text { (the dual-channel option is also used for any other technology } \\
\text { profiling pairs of samples) }\end{array}$ \\
\hline genotype & Genotype information & $\begin{array}{l}\text { A matrix of marker genotypes for each marker and each strain. } \\
\text { The values can be numeric: "I" and "0" for two homozygous } \\
\text { genotypes, respectively (optionally, "0.5" for heterozygous allele). } \\
\text { They can also be characters: "A" "B" or "H" and "H" is for } \\
\text { heterozygous allele; NA for missing data. The column names are } \\
\text { strain names, such as "Strain I", "Strain 2", etc. The row names are } \\
\text { marker names, such as "CIMI", "C2M2", etc. }\end{array}$ \\
\hline
\end{tabular}

nEnvFactors ${ }^{a} \quad$ Number of environmental factors in the study

A numeric integer value between $I$ and 3 which indicates the number of environmental factors to be studied. Experiments with more than three environmental factor are not recommended here since the power to estimate the high-order interactions is very limited for a realistic number of samples (several hundreds).

\begin{tabular}{lll}
\hline nLevels & Number of levels for each environmental factor & $\begin{array}{l}\text { A numeric integer vector. For example, there are two different } \\
\text { levels for two environmental factors under study, then we use } \\
\text { nLevels }<-c(2,2)\end{array}$ \\
\hline Levelb & Level values for each environmental factor & $\begin{array}{l}\text { A list which specifies the levels for each factor in the experiment. } \\
\text { The element is a vector describing all levels of the environmental } \\
\text { factor. In the given example, temperature levels are I6 and } 24 \text { and } \\
\text { drug treatment levels are } 5 \text { and } 10 . \text { The we use: } \\
\text { Level <- } 1 \text { ist (c ( } 16,24), C(5,10))\end{array}$
\end{tabular}

\begin{tabular}{lll}
\hline nSlides & Total number of slides available for the experiment. & A numeric integer value \\
\hline nTuple & $\begin{array}{l}\text { Average number of strains to be assigned onto each } \\
\text { condition }\end{array}$ & A numeric value which is larger than I \\
\hline region & Genome region of biological interest & $\begin{array}{l}\text { A numeric integer vector which indicates the markers of biological } \\
\text { interest, for example those previously detected for phenotypic } \\
\text { quantitative trait loci. The value is the marker index (i.e., the row } \\
\text { number in the genotype data table), not the marker name. }\end{array}$
\end{tabular}

The weights for estimating genetic and environmental
factors, and their interaction terms

A numeric vector which indicates the parameters of biological interest. Higher weights correspond to higher interest, and the optimization is adjusted in such a way as to result in a higher accuracy of the estimate for the parameters with higher weight. Prior knowledge about expected effect sizes of interesting factors can also be incorporated as weight parameters for the algorithm. The weight is inversely proportional to the expected effect size of the corresponding parameter, if the same relative accuracy is intended. When there is no environmental perturbation, weights is I, as there is only one parameter of interest (genotype); When nEnvFactor $=1$, weight $=c\left(w_{Q}\right.$, $\left.\mathrm{w}_{\mathrm{Fl}}, \mathrm{w}_{\mathrm{QFI}}\right)$; When nEnvFactor $=2$, weight $=\mathrm{c}\left(\mathrm{w}_{\mathrm{Q}}, \mathrm{w}_{\mathrm{Fl}}, \mathrm{w}_{\mathrm{F} 2}\right.$, $\left.\mathrm{w}_{\mathrm{QF1}}, \mathrm{W}_{\mathrm{QF2}}, \mathrm{W}_{\mathrm{FIF} 2}, \mathrm{w}_{\mathrm{QFIF} 2}\right)$; When nEnvFactor $=3$, weight $=$

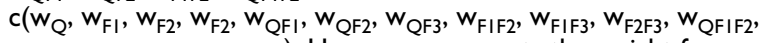
$\left.\mathrm{W}_{\mathrm{QFIF3}}, \mathrm{W}_{\mathrm{QF2F3}}, \mathrm{W}_{\mathrm{QFIF2F3}}\right)$. Here $\mathrm{W}_{\mathrm{Q}}$ represents the weight for genotype effect, $W_{F I}$ represents the weight for environmental factor $F_{1}$ effect and $w_{Q F I}$ represents the weight for interaction between genotype and $F_{1}$ effect, etc. 
Table 2: The description and possible values of designGG arguments (Continued)

\begin{tabular}{lll}
\hline directory $^{b}$ & Output file directory & The path where output files will be saved. \\
\hline fileName & Output file names & The name for output tables in CSV format to be produced.
\end{tabular}

a Required input arguments from users

boptional input arguments

cAlternative arguments: either of them is required

2. Upload a tab separated value (TXT) file containing the genotype data matrix (individuals $\times$ markers). Each cell contains a genotype label (e.g. A or B for the parental alleles, $\mathrm{H}$ for heterozygous loci; NA for missing data).

3. Set parameters. Specify the number of environmental factors, their number of levels, and the possible values of these levels. Specify either the total number of slides (assays) or the number of samples allocated within each condition.

4. Use advanced options if only one or a few genome regions or particular factors are of major interest. It is possible to optimize the experimental design by focusing on certain regions (e.g. the first 20 markers on chromosome I). Prior knowledge about expected effect sizes of interesting factors can also be incorporated as weight parameters for the algorithm.

5. Start the optimization algorithm by clicking on the button Optimize Experimental Design (Figure 1).

6. Get results. After the optimization is finished, the optimal experimental design will be displayed online (in table format), and will be available as text files for download.

\section{R package}

Here we illustrate how to apply the designGG $\mathrm{R}$ package using an example: suppose we are studying the effect of genetic factors $(\mathrm{Q})$, temperature $\left(\mathrm{F}_{1}\right)$, drug treatment $\left(\mathrm{F}_{2}\right)$ and their interaction on gene expression using two-colour microarrays. There are 100 microarray slides available for this experiment, and we plan to study two different levels for each environment, which are $16^{\circ} \mathrm{C}$ and $24^{\circ} \mathrm{C}$ for $\mathrm{F}_{1}$ (temperature), and $5 \mu \mathrm{M}$ and $10 \mu \mathrm{M}$ for $\mathrm{F}_{2}$ (drug treat-

Table 3: Example table of the allocation of strains to arrays.

\begin{tabular}{lll}
\hline & Channel I & Channel 2 \\
\hline array I & Strain 28 & Strain 92 \\
\hline array 2 & Strain 70 & Strain 47 \\
\hline array 3 & Strain 22 & Strain 89 \\
\hline$\ldots$ & $\ldots$ & $\ldots$ \\
\hline
\end{tabular}

This is applicable for technologies that profile samples in pairs, e.g. two-color microarrays. ment). Then the R package can also be used in command line form as follows:

1. Prepare the input file specifying the genotype of each individual at each marker position. The file should be formatted as tab separated values (TXT), as illustrated in Table 1.

2. Load the designGG package by starting the R application and typing the command:

\section{> library (designGG)}

Specify the input arguments (Steps 3-5 correspond to steps 2-4 of using the web tool. The order of the following commands in steps 3-5 does not matter).

3. Choose the platform of the experiment. In this example, we use two-color microarray, thus:

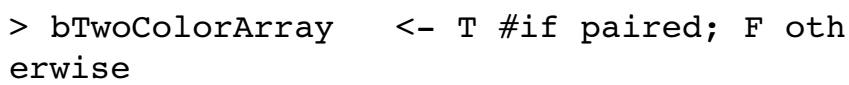
erwise

4. Load the marker data and specify the following required arguments (number of environmental factors, number of levels per factor, the values of each level, and the number of available slides):

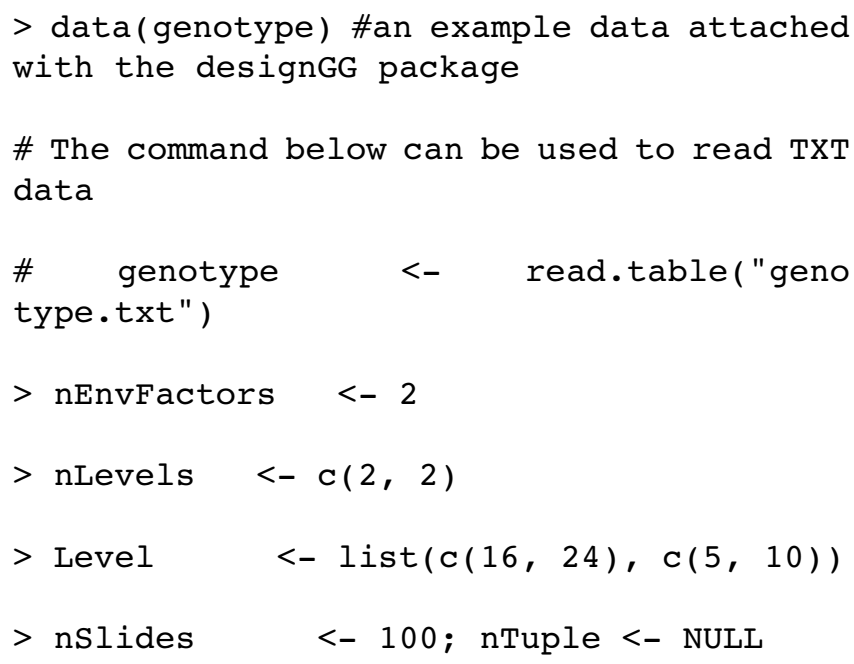

An alternative to specifying nslides is to specify $\mathrm{nTu}$ ple, the number of strains to be allocated onto each condition. For example, 
Table 4: Example table of the allocation of strains to experimental conditions.

\begin{tabular}{llllllll}
\hline & Temperature & Drug & \multicolumn{5}{c}{ Selected Strains } \\
\hline condition I & 16 & 5 & Strain 28 & Strain 81 & Strain 18 & Strain 6I & $\ldots$ \\
\hline condition 2 & 24 & 5 & Strain 70 & Strain 40 & Strain 83 & Strain 92 & $\ldots$ \\
\hline condition 3 & 24 & 10 & Strain 14 & Strain 3 & Strain 89 & Strain 22 & $\ldots$ \\
\hline$\ldots$ & $\ldots$ & $\ldots$ & $\ldots$ & $\ldots$ & $\ldots$ & $\ldots$ \\
\hline
\end{tabular}

If the number of strains is smaller than the number of combinations of factors, the same strain can be used multiple times.

$>$ nTuple <- 25 ; nSlides <- NULL;

5. In addition to the required arguments specified in step 4 , there are some optional ones for a tailored experimental design: e.g., we might be especially interested in the genome region between $1^{\text {st }}$ marker and $20^{\text {th }}$ marker, where a known phenotypic QTL from previous study locates. They can then specify that the optimization algorithm should only take genotypes at markers 1 to 20 into account:

$>$ region $\quad<-\operatorname{seq}(1,20$, by $=1)$

Additionally, if we want that the estimates of all interaction effects are twice as accurate as the estimates of the main effects (genotype, temperature and drug treatment), then we specify weights for the estimates:

$>$ weight $\quad<-c(0.5,0.5,0.5,1,1,1,1)$

Here the order of elements in the weight vector is such that first the main effects are listed, starting with the genotype, followed by the two environmental factors in the order used for nLevels and Level, then the one-way interactions, in the same order, and finally the two-way interaction between all three factors.

6. The following commands specify the directory where the resulting optimal design tables are to be stored and the name of the output files (design tables):

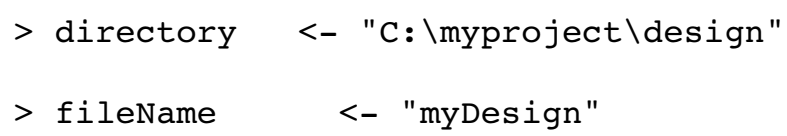

A detailed explanation of the above arguments can also be found in Table 2 .

7. Run designGG to obtain your optimal design:

$>$ myOutput <- designGG(genotype, nSlidesnple, nEnvfactors, nLevels, Levelregiogion, weight $=$ weight, nItera tions $=10$ )
It should be noted that the number of iteration of the simulated annealing method (fnIterations)is set to 10 here for testing purposes. The default value (nItera tions $=3000$ ) is recommended, but it will result in a longer computing time.

8. Output can be found in the directory or retrieved with:

$>\quad$ optimalArrayDesign <- myOut put\$arrayDesign

$>$ optimalCondDesign <- myOutput\$condi tionDesign

Example output tables for allocation of strains on arrays and different conditions are shown in Table 3 and 4, respectively.

9. In addition, users can check the curve of optimization score recorded as the algorithm iterates using:

$>$ plotAllScores (myOutput\$plot.obj)

Details of default settings such as method (SA: simulated annealing) or nSearch (equals 2) can be found in the designGG manual or the online help. Example genotype data and output tables are also provided along with the package. The $\mathrm{R}$ package can be found in Additional file 1 and most up-to-date version of the software can be downloaded at http://gbic.biol.rug.nl/designGG.

\section{Expected Results}

Two tables summarize the optimal design: The table pair design is only used for two-channel experiments and describes how samples are paired together in one assay e.g., a two-color microarray chip (Table 3). The table environment design lists how samples are assigned to environments/experimental factors (Table 4).

\section{Conclusion}

DesignGG, a freely-available R package and web tool presented in this work, represents a novel tool for the researcher interested in system genetics. Based on the care- 
ful experimental design provided by designGG, limited resources, such as arrays and samples, are maximally exploited, and more accurate estimates of parameters of interest can be achieved.

\section{Availability and requiredments}

Project name: designGG R package and web tool

Project home page: http://gbic.biol.rug.nl/designGG

Programming language: $\mathrm{R}$

Requirement: R statistical software available at http:// www.r-project.org/ for the stand-alone version.

\section{Authors' contributions}

YL developed designGG. RCJ and RB directed the project. MAS, GV and JF helped to implement the web tool. All authors wrote the manuscript, and read and approved the final version.

\section{Additional material}

\section{Additional file 1}

designGG: an R-package for the optimal design of genetical genomics experiments. DesignGG aims at finding an optimal design of genetical genomics experiments which maximize the power and resolution of detecting genetic, environmental and interaction effects. This will help to achieve high power and more accurate estimates of the effects of interesting factors, and thus yield a more reliable biological interpretation of data. Click here for file

[http://www.biomedcentral.com/content/supplementary/14712105-10-188-S1.zip]

\section{Acknowledgements}

This work was supported by the Netherlands Organization for Scientific Research, NWO-8650400I. We thank Danny Arends for help in implementing the web tool.

\section{References}

I. Jansen RC, Nap JP: Genetical genomics: the added value from segregation. Trends Genet 200I, I7(7):388-39I.

2. Bystrykh L, Weersing E, Dontje B, Sutton S, Pletcher MT, Wiltshire $\mathrm{T}$, Su Al, Vellenga E, Wang J, Manly KF, et al:: Uncovering regulatory pathways that affect hematopoietic stem cell function using 'genetical genomics'. Nat Genet 2005, 37(3):225-232.

3. Schadt EE, Lamb J, Yang X, Zhu J, Edwards S, Guhathakurta D, Sieberts SK, Monks S, Reitman M, Zhang C, et al: An integrative genomics approach to infer causal associations between gene expression and disease. Nat Genet 2005, 37(7):710-7I7.

4. Chen Y, Zhu J, Lum PY, Yang X, Pinto S, MacNeil DJ, Zhang C, Lamb J. Edwards S, Sieberts SK, et al.: Variations in DNA elucidate molecular networks that cause disease. Nature 2008, 452(7| 86):429-435.

5. Brem RB, Kruglyak L: The landscape of genetic complexity across 5,700 gene expression traits in yeast. Proc Natl Acad Sci USA 2005, 1 02(5): 1572-1577.

6. Li Y, Breitling R, Jansen RC: Generalizing genetical genomics: getting added value from environmental perturbation. Trends Genet 2008, 24(10):518-524.
7. Li Y, Alvarez OA, Gutteling EW, Tijsterman M, Fu J, Riksen JA, Hazendonk E, Prins P, Plasterk RH, Jansen RC, et al.: Mapping determinants of gene expression plasticity by genetical genomics in C. elegans. PLoS Genet 2006, 2(I 2):e222.

8. Churchill GA: Fundamentals of experimental design for cDNA microarrays. Nat Genet 2002, 32(Suppl):490-495.

9. Fu J, Jansen RC: Optimal design and analysis of genetic studies on gene expression. Genetics 2006, 1 72(3): 1993-1999.

10. Fisher RA: The design of experiments. 4th edition. Edinburgh: Oliver and Boyd; 1947.

II. Kerr MK, Churchill GA: Experimental design for gene expression microarrays. Biostatistics 200I, 2(2):183-20I.

12. Yang YH, Speed T: Design issues for cDNA microarray experiments. Nat Rev Genet 2002, 3(8):579-588.

13. Fournier MV, Carvalho PC, Magee DD, Carvalho MGC, Appasani K: Experimental Design for Gene Expression Analysis. In Bioarrays From Basics to Diagnostics Humana Press; 2007:29.

14. Wit E, Nobile A, khanin R: Near-optimal designs for dual-channel microarray studies. Applied Statistics 2005, 54(5):8I7-830.

15. Lam AC, Fu J, Jansen RC, Haley CS, de Koning DJ: Optimal design of genetic studies of gene expression with two-color microarrays in outbred crosses. Genetics 2008, 180(3):169|-1698.

16. Rosa G], de Leon N, Rosa AJ: Review of microarray experimental design strategies for genetical genomics studies. Physiol Genomics 2006, 28(I): 15-23.

17. Woo Y, Krueger W, Kaur A, Churchill G: Experimental design for three-color and four-color gene expression microarrays. Bioinformatics 2005, 2 I (Suppl I): $4459-467$.

18. Wit E, Nobile A, Khanin R: Simulated annealing for near-optimal dual-channel microarray designs. Appl Statistics 2005:817-830

19. The R Project for Statistical Computing [http://www.rproject.org/]

20. Swertz MA, De Brock EO, Van Hijum SA, De Jong A, Buist G, Baerends RJ, Kok J, Kuipers OP, Jansen RC: Molecular Genetics Information System (MOLGENIS): alternatives in developing local experimental genomics databases. Bioinformatics 2004, 20(13):2075-2083.

21. Swertz MA, Jansen RC: Beyond standardization: dynamic software infrastructures for systems biology. Nat Rev Genet 2007, 8(3):235-243.

\section{Publish with Bio Med Central and every scientist can read your work free of charge}

"BioMed Central will be the most significant development for disseminating the results of biomedical research in our lifetime. "

Sir Paul Nurse, Cancer Research UK

Your research papers will be:

- available free of charge to the entire biomedical community

- peer reviewed and published immediately upon acceptance

- cited in PubMed and archived on PubMed Central

- yours - you keep the copyright
BioMedcentral 